\title{
Data analysis at Level-1 Trigger level
}

\author{
Johannes Wittmann*', Bernhard Arnold, Gregor Aradi, Herbert Bergauer, Manfred \\ Jeitler and Claudia-Elisabeth Wulz \\ Austrian Academy of Sciences, Institute of High Energy Physics \\ E-mail: Johannes.Wittmann@cern.ch, Bernhard. Arnold@oeaw.ac.at, \\ Gregor.Aradidoeaw.ac.at, Herbert.Bergauerdoeaw.ac.at, \\ Manfred.Jeitler@cern.ch, Claudia.Wulz@cern.ch
}

\section{Leonard Apanasevich}

University of Illinois at Chicago

E-mail: Leonard.Apanasevich@cern.ch

\section{Brian L. Winer and Darren M. Puigh}

Ohio State University

E-mail: Brian.Winer@cern.ch, dpuigh@gmail.com

\begin{abstract}
With ever increasing luminosity at the LHC, optimum online data selection is getting more and more important. While in the case of some experiments (LHCb and ALICE) this task is being completely transferred to computer farms, the others - ATLAS and CMS - will not be able to do this in the medium-term future for technological, detector-related reasons. Therefore, these experiments pursue the complementary approach of migrating more and more of the offline and High-Level Trigger intelligence into the trigger electronics. This paper illustrates how the Level-1 Trigger of the CMS experiment and in particular its concluding stage, the Global Trigger, take up this challenge.
\end{abstract}

Topical Workshop on Electronics for Particle Physics 11 - 14 September 2017

Santa Cruz, California

\footnotetext{
${ }^{*}$ Speaker.

${ }^{\dagger}$ for the CMS collaboration
} 


\section{Introduction}

The CMS detector uses a two level trigger system for online data selection. The first level of this system (the Level-1 Trigger) is implemented in hardware. The second level, the High-Level Trigger (HLT), is implemented in software [1]. While during the early years of LHC operation the Level-1 Trigger selection was dominated by single-object triggers whose thresholds (in particular, thresholds on transverse momentum or transverse energy) were gradually raised to limit trigger rates to manageable levels, higher luminosity and higher pile-up would now result in significant loss of good physics data and is not viable any more. So, while silicon-tracker information will still be available only at the HLT for a number of years to come, the Level-1 Trigger relies increasingly on topological combinations of trigger objects from calorimeters and muon systems and on physics quantities such as invariant mass or transverse mass [2]. This requires using particle flight directions correctly extrapolated to the vertex rather than raw detector data distorted by the bending of charged-particle tracks in the magnetic field. The enormous progress in digital electronics, in particular with regard to the computing power of Field-Programmable Gate Arrays (FPGAs) makes it possible to use input data of higher resolution and to perform complex calculations using Digital Signal Processors (DSPs) as well as big lookup tables for large numbers of candidate objects. The available resources also allow us to calculate in parallel certain quantities such as missing transverse energy (missing transverse momentum) in different ways (integrating over different ranges in pseudorapidity) and use for each physics channel the most adequate version of this quantity. As hadronic jets, electron/photon candidates and "hadronic tau decays" (narrow jets from hadronic tau lepton decays) can be discriminated in the calorimeters only to a limited extent, attention must be paid to avoid double counting of such objects. By better reflecting HLT resolution and algorithms in the Level-1 Trigger electronics, a high level of efficiency can be maintained and more useful physics events can be obtained within the available bandwidth [3].

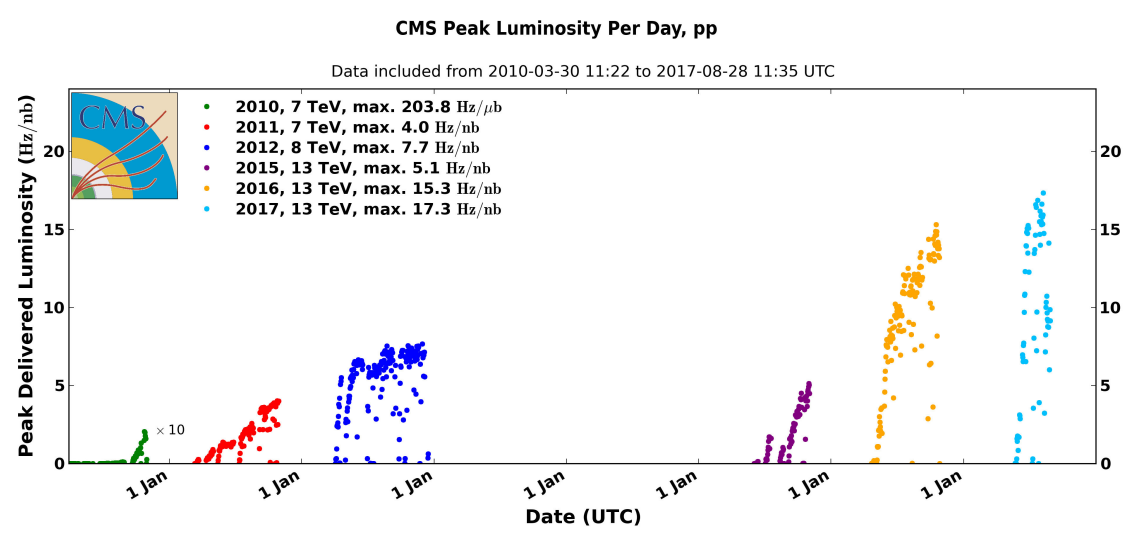

Figure 1: Peak Luminosity versus time for 2010 - 2012 and 2015 - 2017 (p-p data only) [4].

\section{New challenges for the Level-1 Trigger}

The Level-1 Trigger of the CMS experiment is facing new challenges because of the impressive performance of the LHC. This has resulted in a luminosity of more than $1.7 \times 10^{34} \mathrm{~cm}^{-2} \mathrm{~s}^{-1}$ 
(see Figure 1) and a pile-up (proton collisions during one bunch crossing) of more than 45 superimposed events. New techniques and algorithms need to be developed to get better online data reduction. A transfer of data analysis methods to the Level-1 Trigger can help to achieve this goal. Figure 2 shows the Level-1 Trigger system with its concluding stage - the Global Trigger - marked with a red circle.

\section{The CMS Global Trigger and its features}

The Global Trigger (GT) combines information from the muon and the calorimeter trigger paths together with binary inputs from other sources (such as the LHC beam detector "BPTX" and others) [5]. The GT implements a variety of complex physics algorithms, which together are called the Level-1 menu. In 2017, the system reached its full capability and is now using six MP7 modules (using powerful XILINX Virtex-7 FPGAs [6]) running in parallel to provide enough logic resources for implementing all desired physics algorithms. The number of these trigger algorithms was limited to 128 in the past and has been increased to 512 with the commissioning of the upgraded Global Trigger. The development of the maximum number of physics algorithms implemented in the Level-1 menu for the last 3 years is shown in Figure 3. To assist the user in putting together Level-1 menus, the Trigger Menu Editor (TME) software and its specially designed, flexible grammar have been developed. Combining the trigger menu with predefined, static VHDL templates guarantees a fast and reliable generation of the Global Trigger firmware [7]. Prescales are used to reduce the trigger rate of algorithms. For example: a prescale value of 2 halves the rate of an algorithm. A new feature introduced in 2017 is the "prescale preview" functionality: a second set of prescales can be selected in parallel during data taking and the expected trigger rates for these prescales are calculated. A dedicated AMC502 [8] prescale preview module facilitates applying the correct prescale values. Figure 4 shows the fully equipped Global Trigger crate in operation at CMS since 2017. The left side holds the six MP7 modules, which have the algorithms implemented. The right part holds six AMC502 boards built from Vadatech used for generating the final trigger signal, calculating the preview prescale rates and receiving the external binary inputs.

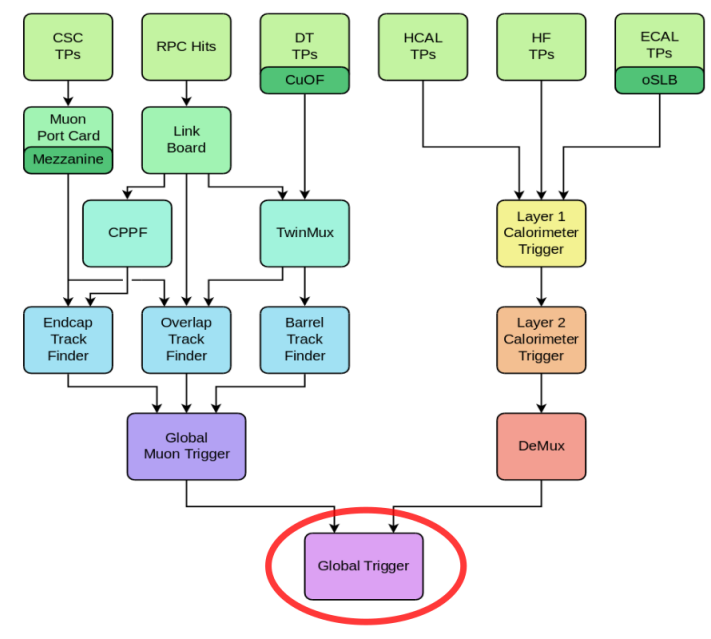

Figure 2: The Level-1 Trigger system [9].

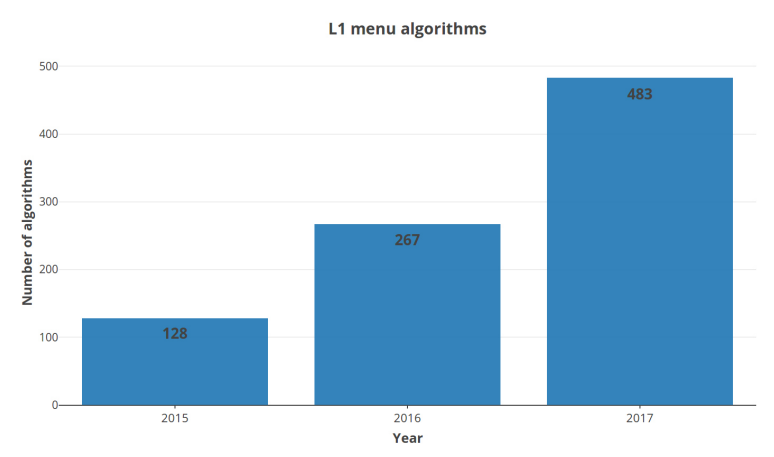

Figure 3: Maximum number of algorithms used in the Level-1 Menu from 2015-2017. 


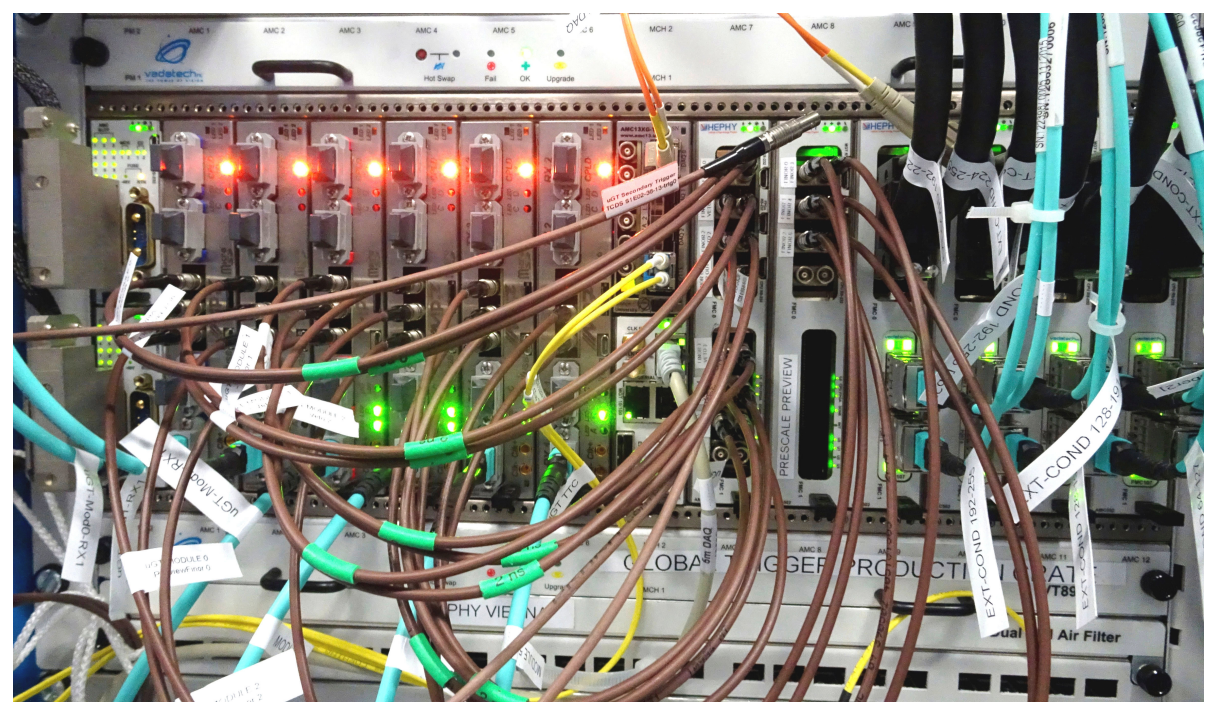

Figure 4: The fully equipped Global Trigger crate.

\section{New Global Trigger Algorithms}

Several new types of trigger algorithms are migrating complex selection requirements from offline analysis and HLT to the Level-1 Trigger electronics. New techniques will help keeping the Level-1 acceptance rate below $100 \mathrm{kHz}$, which is the maximum output rate. In addition to the invariant-mass algorithms, new transverse-mass triggers for objects without pseudorapidity information have been introduced. Mass triggers and other algorithms now have an optional "two-body pt" cut, which is the transverse momentum calculated for the hypothetical mother particle of the two trigger objects whose mass is being calculated. A new feature makes it possible to add constraints on the number of objects used by an algorithm. The index of objects in a collection can be confined to accept, e.g., just the leading jet of an event. Also a combination of different indices can be used to combine, e.g., the leading jet of an event with the leading four electron/gamma candidates. Apart from making finer object selection possible, this feature is also a useful way to save FPGA resources. Another important issue is to avoid double counting of calorimeter objects in complex trigger algorithms (e.g. the same particle is seen as jet and as tau) by introducing a function called "Overlap Remover". This new function allows to define a window around a calorimeter object, in which no other calorimeter objects are considered in the algorithm processing.

An example of the migration of complex selection algorithms from offline analysis to the Level-1 Trigger electronics is a new Level-1 Trigger for Vector Boson Fusion processes (VBF - see Figure 5). VBF is an important channel in the $H \rightarrow \tau \tau$ analysis. Two quarks emit virtual vector bosons, which fuse and create a Higgs boson. The jets from the surviving quarks usually subtend a large angle and can be efficiently selected by applying a lower cut on the invariant-mass calculated by $M_{i n v} \approx \sqrt{2 p_{T 1} p_{T 2} \times\left[\cosh \left(\eta_{1}-\eta_{2}\right)-\cos \left(\varphi_{1}-\varphi_{2}\right)\right]}$. This mass cut can also be combined with other conditions to find the Higg's decay products (such as muons). 


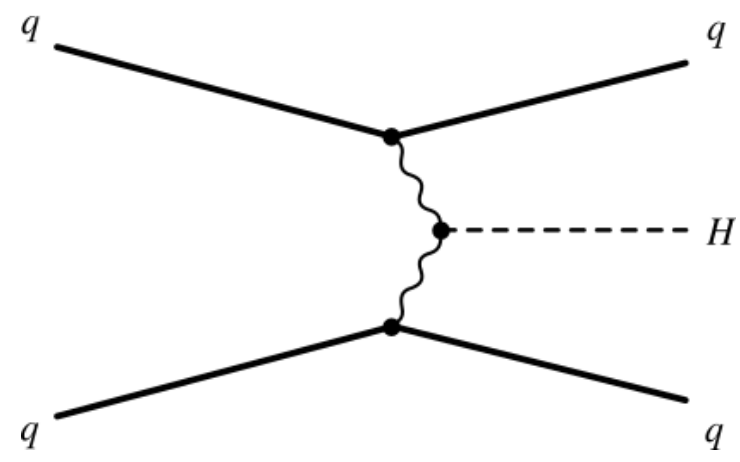

Figure 5: Feynman diagram showing Higgs production through VBF.

\section{Conclusion}

The impressive performance of the LHC accelerator is a challenge for the Level-1 Trigger. The techniques presented and new types of algorithms help keep the trigger rates at a manageable level without increasing thresholds in transverse momentum to a point that would result in significant loss of physics data. To keep pace with the further improvements in LHC performance, continuous development of better selection algorithms in the Global Trigger is required.

\section{References}

[1] V. Khachatryan et al., The CMS trigger system, Journal of Instrumentation Vol 12, No 1, p P01020 (2017)

[2] A. Tapper and D. Acosta, CMS Technical Design Report for the Level-1 Trigger Upgrade, CERN-LHCC-2013-011. CMS-TDR-12 (2013)

[3] M. Jeitler et al., The upgrade of the CMS trigger system, Journal of Instrumentation Vol 9, No 8, p C08002 (2014)

[4] https://twiki.cern.ch/twiki/bin/view/CMSPublic/LumiPublicResults, CMS Collaboration (04.09.2017)

[5] J. Wittmann et al., Design and performance of the phase I upgrade of the CMS Global Trigger, Journal of Instrumentation Vol 12, No 1, p C01046 (2017)

[6] A. Rose et al., The MP7 and CTP-6: multi-hundred Gbps processing boards for calorimeter trigger upgrades at CMS, Journal of Instrumentation Vol 7, No 12, p C12024 (2012)

[7] T. Matsushita and B. Arnold, Software for implementing trigger algorithms on the upgraded CMS Global Trigger System, Journal of Physics: Conference Series Vol 664, No 8, p 082031 (2015)

[8] AMC502 product page, http://www.vadatech.com/product.php?product=396, Vadatech Incorporated.

[9] https://treis.web.cern.ch/treis/cms/11_muon_upgrade/docs/, Thomas Reis (10.10.2017) 